\title{
DEFORMATION OF FE3SI SINGLE-CRYSTALS UNDER NANOINDENTATION
}

\author{
Jaroslav Čech*, Petr Haušild, Aleš Materna \\ Czech Technical University in Prague, Faculty of Nuclear Sciences and Physical Engineering, Trojanova 13, \\ 12000 Praha, Czech Republic \\ * corresponding author: jaroslav.cech@fjfi.cvut.cz
}

\begin{abstract}
Knowledge of the complex deformation behavior in the anisotropic materials is one of essential issues in materials science and it is crucial for the applications of a given material. In this study, mechanical response of Fe3(wt.\%)Si single crystal to nanoindentation with spherical indenter was investigated. Hardness and indentation Young's modulus were determined experimentally and by finite element modelling. Observed pop-in phenomenon, shape of the residual imprints and origin of the slip lines were explained on the basis of resolved shear stress computed by finite element model.
\end{abstract}

KEYWORDS: nanoindentation, bcc, slip activation, mechanical properties, finite element model.

\section{INTRODUCTION}

Nanoindentation is a very useful tool for characterization of mechanical properties of various materials in micro(nano)-volume. Nowadays, it is still more and more widespread method as the preparation of the specimens is not difficult and the amount of the material needed for testing is low. It gives the information about basic material properties, e.g. hardness, Young's modulus or fracture toughness. Nanoindentation is a well-established and normalized method [1] and basic procedures of data analysis are already implemented in commercial devices.

More profound analysis of the entire indentation test including the investigation of force-depth curves and residual imprints together with finite element modelling can result in complex description of deformation behavior of the materials under the examination. These detailed studies are usually necessary when investigating the anisotropic materials with crystallographic structure. Many models based on the finite element (FE) method [2 [5] or molecular dynamics [6, 7] were recently developed to describe the distribution of stresses and strains under the indenter. They provide not only the information about material properties, but they can also describe the evolution and propagation of defects (e.g. dislocations, twinning) in the studied material.

In this paper, deformation in different orientations of body centered cubic (bcc) Fe3(wt.\%)Si single crystal was investigated. Nanoindentation was used to characterize the changes in mechanical properties with crystallographic orientation. Finite element model was developed to describe the stress field under the indenter and to explain the differences in the shape of residual imprints.

\section{Experimental Details}

The single crystals of bcc Fe3(wt.\%)Si were produced by the floating-zone melting technique. The material was chosen for this study as its properties depend on the crystallographic orientation [8, 9] and it is often taken as a model material of ferritic steels and iron based alloys.

Tests were performed on the samples oriented with the directions [100] and [110] perpendicular to the specimen surface (Figures 1 and 2). Surface was prepared by standard metallographic procedures (mechanical gridding and polishing up to $1 \mu \mathrm{m}$ diamond paste) and finished by electrolytic polishing to remove residual stresses in the surface layers.

Nanoindentation tests were carried out on NHT2 nanoindentation instrument (Anton Paar, Graz) with diamond spherical indenter with nominal radius $20 \mu \mathrm{m}$. Maximum loading force $75 \mathrm{mN}$ was reached after $30 \mathrm{~s}$. Loading sequence was followed by $10 \mathrm{~s}$ of the holding at maximum load. Unloading time was the same as for loading, i.e. $30 \mathrm{~s}$. To obtain values of hardness and indentation Young's modulus, data were treated by Oliver-Pharr method [10, 11]. Poisson's ratio was supposed 0.3. In order to enable the observation of activated slip systems and residual imprints by means of optical and scanning electron microscopy, indentations to maximum force $500 \mathrm{mN}$ were performed.

FEM nonlinear code MSC.Marc was used for numerical evaluation of the stress field under the indenter. The contact between the rigid indenter and deformable material was considered frictionless due to very low tangential angle between sample and indenter tip. The finite element mesh of indented material consisted of 343500 eight-node linear brick elements and 35 sixnode linear wedge elements placed directly under the indenter center (Figure 31). The smallest elements with a size approximately $0.1 \mu \mathrm{m}$ were in the area of the supposed contact and its close surroundings. The entire model has the diameter of about $1000 \mu \mathrm{m}$ 

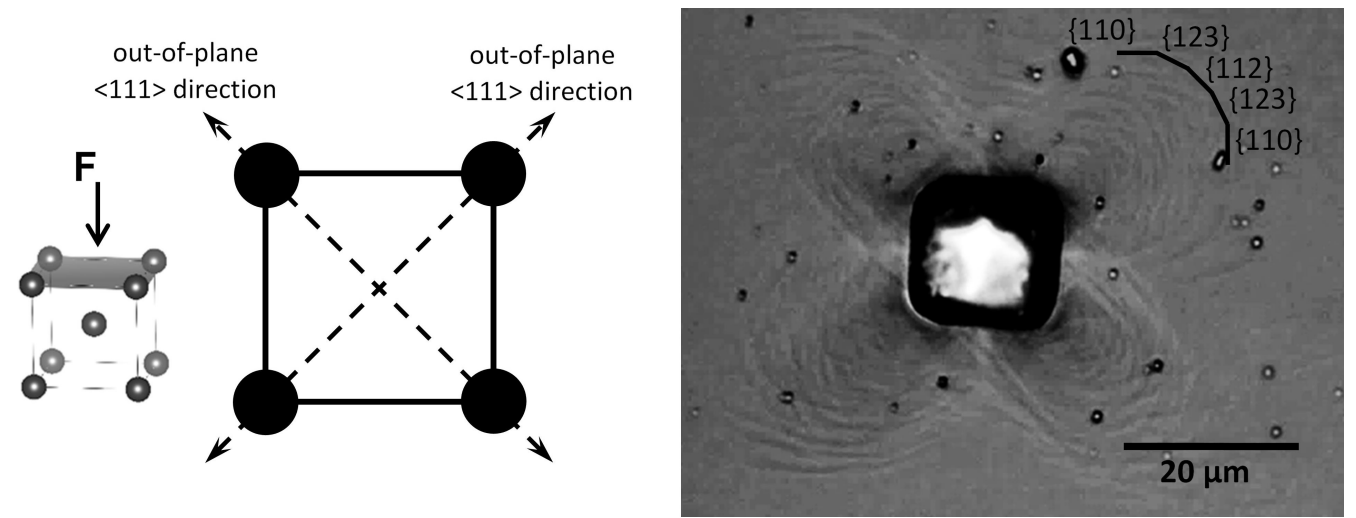

FiguRE 1. Orientation of atoms in the plane parallel with the surface and residual imprint with shown slip systems for [100] crystal orientation.

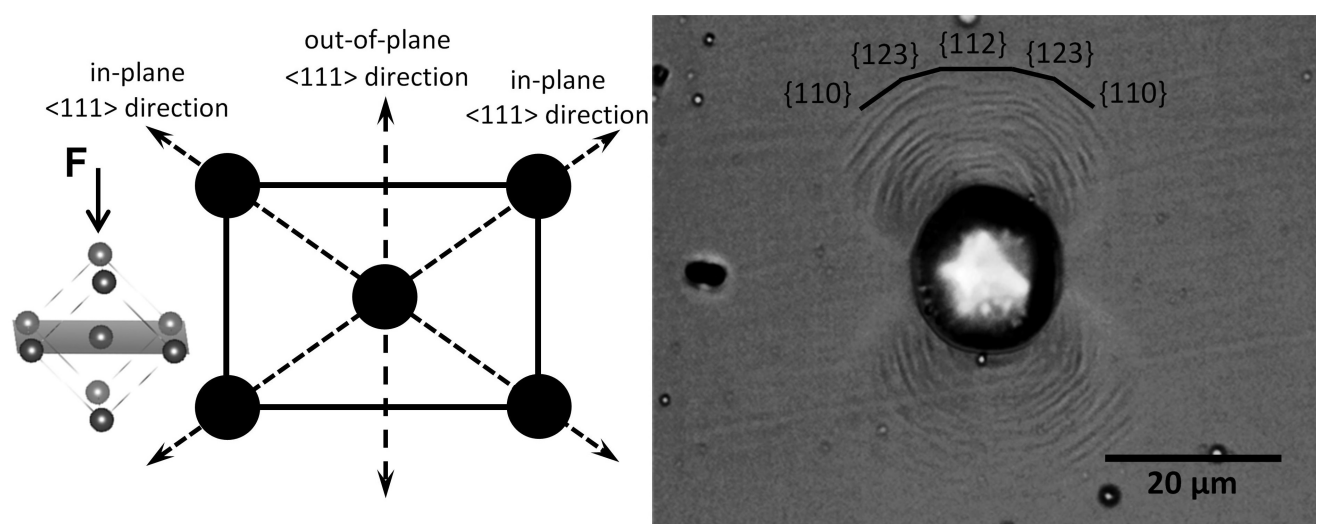

FIgURE 2. Orientation of atoms in the plane parallel with the surface and residual imprint with shown slip systems for [110] crystal orientation.

and height of about $500 \mu \mathrm{m}$. The specimen was fixed only on its bottom, as the relatively large size of the specimen minimized the effect of boundary conditions on its exterior on the achieved results.

Elastic constants used in the model were taken from ref. [12] as: $c_{11}=222.3 \mathrm{GPa}, c_{12}=135.5 \mathrm{GPa}$, and $c_{44}=123.0 \mathrm{GPa}$. Resolved shear stress $\tau_{R S S}$ corresponding to all possible slip system for bcc structure (i.e. 48 slip systems for planes $\{110\},\{112\},\{123\}$ and directions $<111>$ ) was computed using Schmid 's law in the form for multiaxial stress state:

$$
\begin{aligned}
\tau_{R S S} & =l_{n x} l_{d x} \tau_{x x}+l_{n y} l_{d y} \tau_{y y}+l_{n z} l_{d z} \tau_{z z}+ \\
& +\left(l_{n y} l_{d x}+l_{n x} l_{d y}\right) \tau_{x y}+\left(l_{n z} l_{d y}+l_{n y} l_{d z}\right) \tau_{y z}+ \\
& +\left(l_{n x} l_{d z}+l_{n z} l_{d x}\right) \tau_{z x}
\end{aligned}
$$

where $l$ are direction cosines between the axes and slip direction $d$ or normal to the slip plane $n$ and $\tau_{i j}$ is stress tensor.

\section{Results}

Experimental indentation curves showing the dependence of applied force on penetration depth are in Figure 4. For both orientations, the same behavior was observed. Sudden increases in penetration depth at constant load called pop-ins were spread through whole range of applied forces. For some curves the popin did not occur even for the maximum applied load of $75 \mathrm{mN}$ and these curves were completely elastic, which was confirmed by zero residual depth and no residual imprint after the unloading. On the other hand, the curves with nearly immediate development of plastic deformation were also observed. In Figure 4 4 , elastic and "completely plastic" curves are compared. It can be seen that both type of curves for orientation [110] are stiffer than for orientation [100].

Hardness and indentation Young's modulus, measured experimentally and computed by finite element model are summarized in Table 11. Hardness evaluated from the indentation curves with developed plastic deformation was approximately $1.7 \mathrm{GPa}$ and it was slightly higher for orientation [110] than [100]. The differences in indentation Young's modulus were more pronounced. Both approaches, experiments and finite element modeling, showed that orientation [110] is stiffer than orientation [100].

Shape of residual imprints made by indentation to maximum force $500 \mathrm{mN}$ is shown in Figures 1 and 2 For orientation [100] significant deviation from expected spherical shape was observed. The deformation spreads preferably in the directions of diagonals of the cubic lattice in the dense directions $<111>$ creating characteristic four-fold symmetry of the imprint. The 

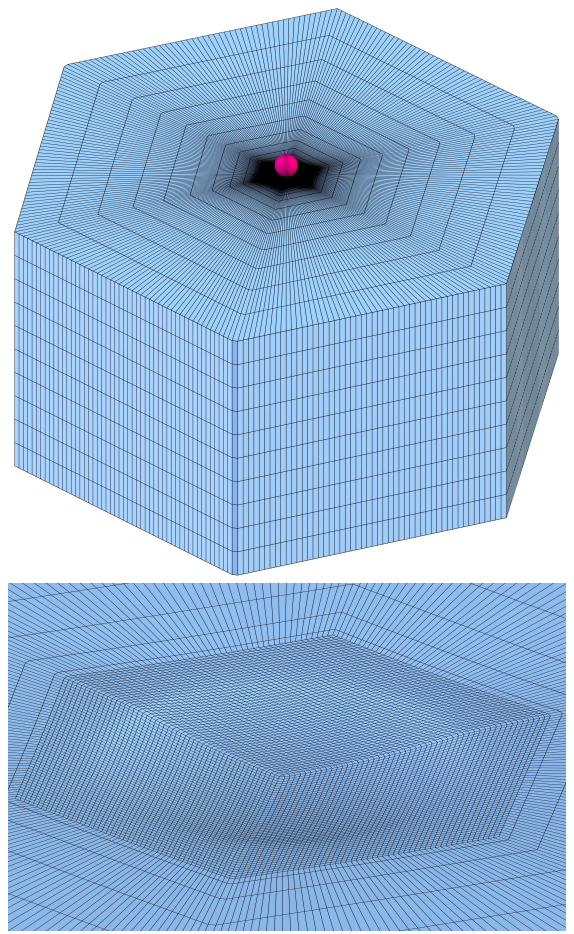

Figure 3. Finite element mesh.

shape of the residual indent for orientation [110] is more regular. The spherical imprint is only slightly elongated into the ellipsoidal shape with two-fold symmetry. For both orientations, intersections of slip lines with the surface are clearly visible.

Resolved shear stress in the available slip systems for bcc structure were computed and the critical slip systems with their maxima were identified by finite element analysis (Table 1). Example of the distribution of the resolved shear stress is shown in Figure 5 . For both orientations, highest values of resolved shear stress were in $\{112\}$ planes. Only slightly lower values were computed for the planes $\{123\}$ and the lowest resolved shear stress acts in planes $\{110\}$. In all critical systems, maximum shear stress was identified for $<111>$ out-of-plane directions (i.e. $<111>$ directions not located in the surface layer). Differences between two studied orientations were found in the values of the maximum resolved shear stress and in the number of available slip systems. While the maximum of the resolved shear stress is approximately 1.5times higher for orientation [110], the number of the slip systems with maximum resolved shear stress is two times lower for this orientation than for the orientation [100].

\section{Discussion}

Generally, pop-in behavior can be related to several phenomena in the material (e.g. film rupture, beginning of plasticity). As, in the case of the studied material, plastic deformation did not occur before the pop-in event, it is unambiguous that the observed popins are associated with the activation of slip and/or nucleation of dislocations with consequent develop-
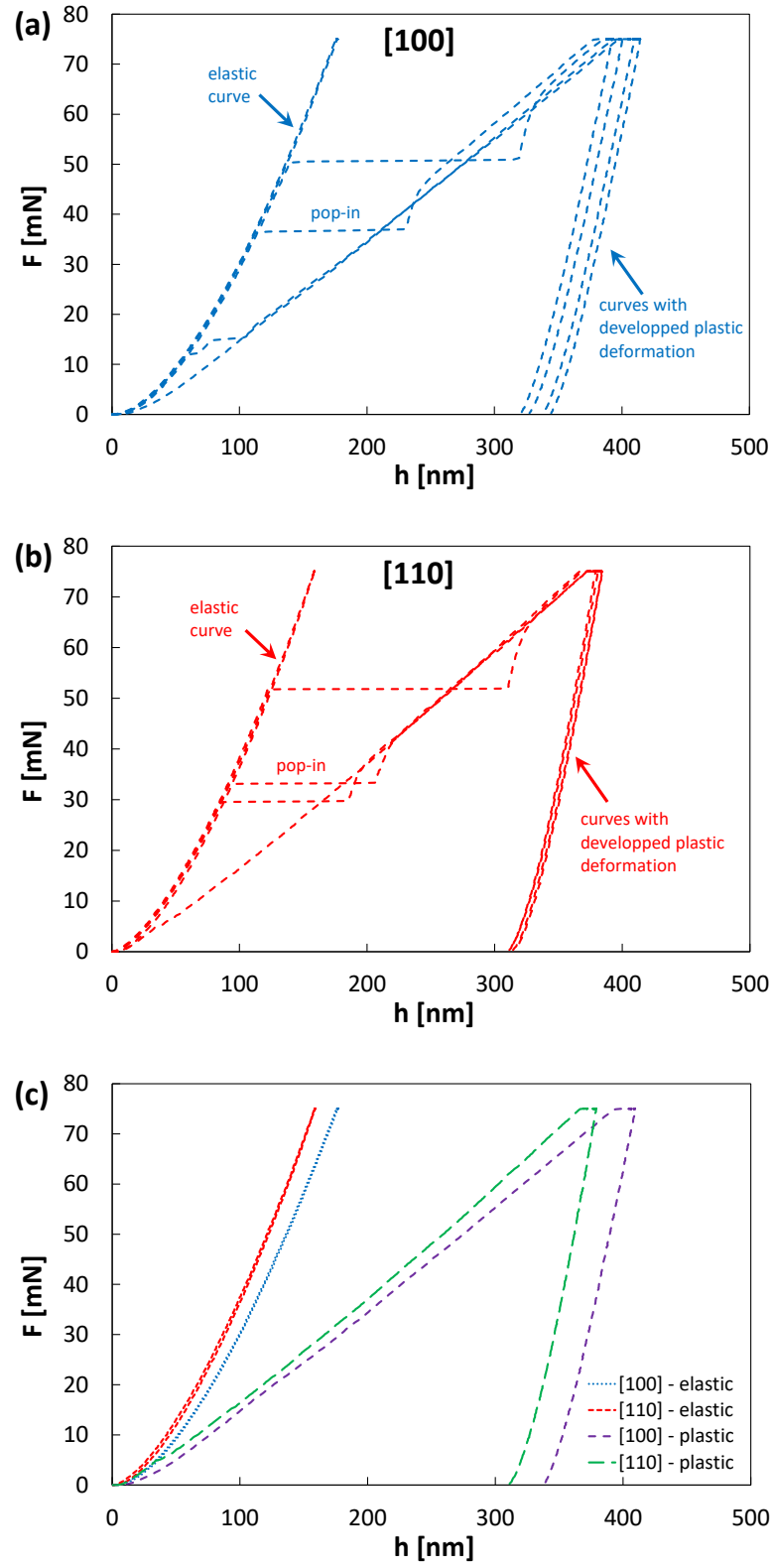

FiguRE 4. Experimental indentation curves for (a) [100] orientation, (b) [110] orientations and (c) comparison of elastic curves and plastic curves with nearly immediate development of plasticity after the contact for both orientations.

ment of plastic deformation.

The spread in the force for which the pop-in event occurs is caused by the distribution of pre-existing dislocations in the material and the position of the maximum of resolved shear stress under the indenter. The shear stress is not symmetric (Figure 5) and its maximum is located in depth under the surface in a different positions depending on the slip system (Figure 6). If the maximum is near the pre-existing dislocation, only low value of stress is needed for the activation of the slip and the plastic deformation starts nearly immediately after the contact of the indenter with the surface. This value of shear stress should correspond to the values of critical resolved shear 


\begin{tabular}{|c|c|c|c|c|c|c|}
\hline orientation & $\boldsymbol{H}[\mathrm{MPa}]$ & $E_{E X P}[\mathrm{GPa}]$ & $E_{F E}[\mathrm{GPa}]$ & slip plane & $\begin{array}{c}\tau_{R S S}[\mathrm{MPa}] \\
\left(F_{\max }=75 \mathrm{mN}\right)\end{array}$ & $\begin{array}{c}\text { No. of equivalent slip systems } \\
\text { with maximum } \tau_{R R S}\end{array}$ \\
\hline & & & & $\{110\}$ & 2827 & 8 \\
\hline \multirow[t]{3}{*}[100]{} & $1698 \pm 55$ & $183.0 \pm 4.5$ & 202.6 & $\{112\}$ & 2905 & 4 \\
\hline & & & & $\{123\}$ & 2893 & 8 \\
\hline & & & & $\{110\}$ & 4269 & 4 \\
\hline \multirow[t]{2}{*}{ [110] } & $1754 \pm 10$ & $221.2 \pm 5.7$ & 229.3 & $\{112\}$ & 4634 & 2 \\
\hline & & & & $\{123\}$ & 4587 & 4 \\
\hline
\end{tabular}

TABLE 1. Mechanical properties and resolved shear stress in available slip systems for orientations [100] and [110] of Fe3Si single crystal.

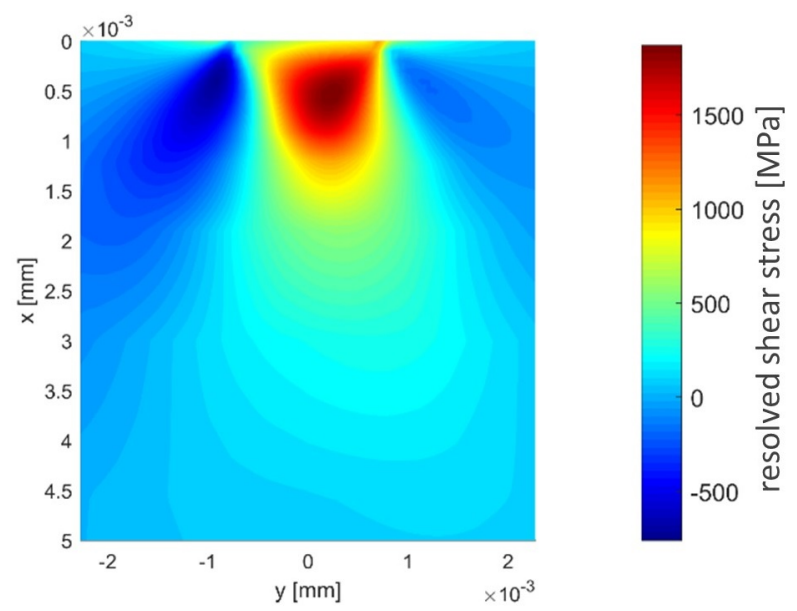

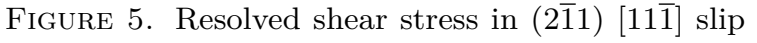
system for the crystal in orientation [100] (cut in (011) plane).

stress for uniaxial tensile tests which is in the range of hundreds of $\mathrm{MPa} 13$. On the other hand, if there is no dislocation near the maximum of the resolved shear stress, significantly higher stress is needed for homogeneous dislocation nucleation. This stress is for $\mathrm{Fe} 3($ wt.\%)Si defect-free single crystal in the range of $G / 20$ [14], where $G$ is the shear modulus. This value is comparable (for the orientation [110]) or even higher (for the orientation [100]) than the values of resolved shear stress computed for maximum load $75 \mathrm{mN}$. It explains the existence of the indentation curves which are completely elastic. Thus, the majority of popins spread through the whole range of applied loads corresponds to the activation of the slip on pre-existing dislocations located near the maximum of the resolved shear stress.

The values of indentation Young's modulus determined experimentally are slightly lower than the values from FE model. Besides the values of elastic constants used in the model which can be slightly different than the values taken from the literature, it is probably caused by the shape of the residual imprint. In elastic FE model, imprint is only slightly deformed as can be seen in Figure 6. On the other hand, during the experiment, indentation Young 's modulus is determined at the beginning of the unloading [10] when the shape of the imprint can significantly deviate from the ideal elastic shape (Figures 1 and 2). Consequently, the contact area can be different which leads to mismatch in measured and modeled indentation Young's modulus. Another reason causing the difference in the shape of the experimental and modeled imprint (and consequently in the indentation Young's modulus) is the shape of the indenter itself [15]. Imperfect shape of the spherical indenter leads to inaccurate determination of actual contact area and it can have effect on the resulting values of mechanical properties.

The shape of the residual imprints can be explained based on activated slip systems whose intersections with the surface can be seen near the residual imprint on the surface. The intersections of the active planes determined by FE model correspond well to these slip lines for both material orientations (Figures 1 and 2 . For orientation [100], the number of active slip system is two times higher than for orientation [110]. This amount is sufficient for the development of deformation in the preferred $<111>$ out-of-plane directions and the shape of the residual imprint consequently deviates from the spherical shape. For orientation [110] there is not enough of slip systems which enables the deformation only in $<111>$ out-of-plane directions. Nevertheless, stresses in other slip systems are high enough to facilitate the deformation in other systems and directions, which leads to more spherical shape of the residual imprint.

\section{Conclusions}

Mechanical properties and deformation behavior of Fe3(wt.\%)Si single crystals under spherical indenter were investigated in this study. It was found that orientation [110] is stiffer and slightly harder than orientation [100]. Pop-in behavior was observed for both orientations. Based on the resolved shear stress computed by finite element method, it was found that most of the pop-ins is caused by activation of slip on pre-existing dislocations. Four-fold symmetry of the 

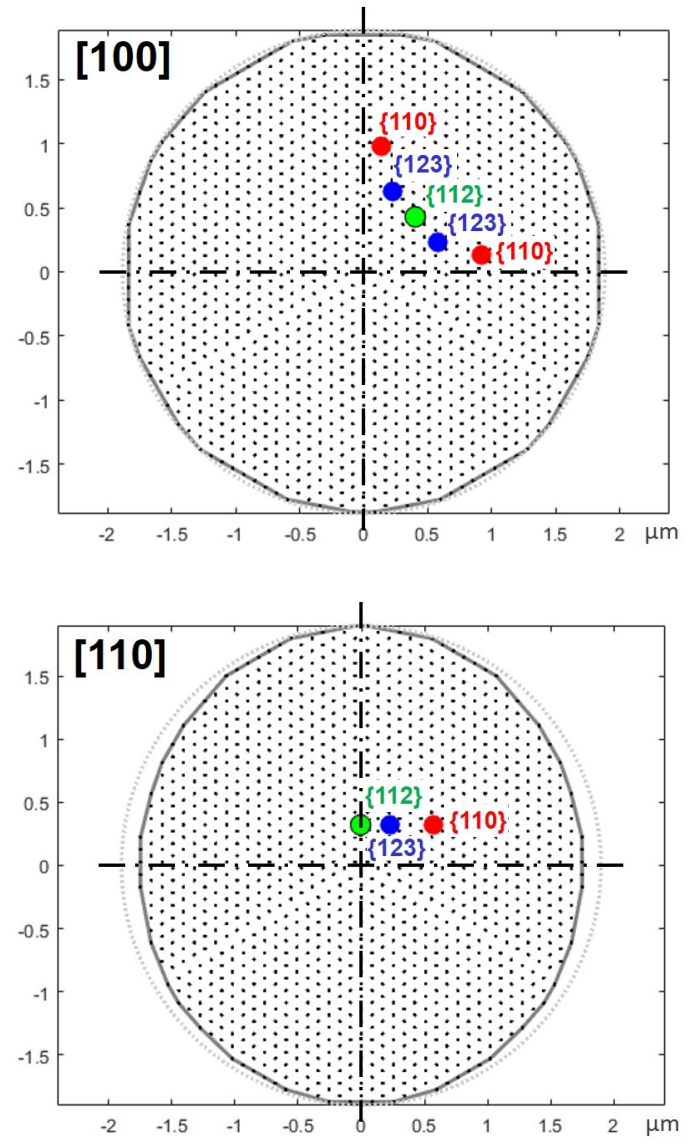

FiguRE 6. Location of the maximum of resolved shear stress under the indenter for critical slip systems (only one quarter of slip systems is displayed because of the symmetry).

residual imprint for the crystal orientation [100] and two-fold symmetry for orientation [110] was explained by the different activation of slip systems. Description of deformation behavior of this anisotropic material with bcc structure can be helpful in the future research and data interpretation of more complex systems.

\section{ACKNOWLEDGEMENTS}

This research was carried out in the frame of the projects 14-36566G and 17-07559S (Czech Science Foundation).

\section{REFERENCES}

[1] ISO 14557, Metallic materials - Instrumented indentation test for hardness and material parameters, 2002.

[2] V. Gaspard, G. Kermouche, D. Delafosse,

A. Barnoush. Hydrogen effect on dislocation nucleation in a ferritic alloy $\mathrm{Fe}-15 \mathrm{Cr}$ as observed per nanoindentation. Mater Sci Eng A 604:86-91, 2014. DOI:10.1016/j.msea.2014.02.041

[3] L. Ma, D. J. Moris, S. L. Jennerjohn, et al. The role of probe shape on the initiation of metal plasticity in nanoindentation. Acta Mater 60:4729-4739, 2012. DOI:10.1016/j.actamat.2012.05.026

[4] A. Materna, P. Haušild, J. Nohava. A numerical investigation of the effect of cubic crystals orientation on the indentation modulus. Acta Phys Pol A 128:693-696, 2015. DOI:10.12693/APhysPolA.128.693

[5] F. Siska, T. Guo, L. Stratil, et al. Numerical study of stress distribution and size effect during AZ31 nanoindentation. Comput Mater Sci 126:393-399, 2017. DOI:10.1016/j.commatsci.2016.10.012

[6] R. Smith, D. Christopher, S. D. Kenny. Defect generation and pileup of atoms during nanoindentation of Fe single crystals. Phys Rev B 67:245405:1-10, 2002. DOI:10.1103/PhysRevB.67.245405

[7] M. A. Lodes, A. Hartmaier, M. Goken, K. Durst. Influence of dislocation density on the pop-in behavior and indentation size effect in $\mathrm{CaF}_{2}$ single crystals: Experiments and molecular dynamics simulations. Acta Mater 59:4264-4273, 2011. DOI:10.1016/j.actamat.2011.03.050

[8] J. Prahl, A. Machová, M. Landa, et al. Fracture of Fe-3 wt.\% Si single crystals. Mater Sci Eng A 462:178-182, 207. DOI:10.1016/j.msea.2006.02.456

[9] A. Spielmannová, M. Landa, P. Machová, A. Haušild, P. Lejček. Influence of crack orientation on the ductile-brittle behavior in Fe-3 wt.\% Si single crystals. Mater Charact 58:892-900, 2007. DOI:10.1016/j.matchar.2006.09.001

[10] W. C. Oliver, G. M. Pharr. An improved technique for determining hardness and elastic modulus using load and displacement sensing indentation experiments. J Mater Res 7:1564-1583, 1992. DOI:10.1557/JMR.1992.1564

[11] W. C. Oliver, G. M. Pharr. Measurement of hardness and elastic modulus by instrumented indentation: Advances in understanding and refinements to methodology. J Mater Res 19:3-20, 2004. DOI:10.1557/jmr.2004.19.1.3

[12] A. Machová, S. Kadečková. Elastic constants of iron-silicon alloy single crystals. Czech J Phys B 27:555-563, 1977. DOI:10.1007/BF01587133

[13] J. Prahl. Deformace a lom monokrystalů Fe-3hm.\%Si: Studie na více úrovních. PhD thesis p. 140, 2008.

[14] A. Barnoush, H. Vehoff. Recent developments in the study of hydrogen embrittlement: Hydrogen effect on dislocation nucleation. Acta Mater 51:5274-5285, 2010. DOI:10.1016/j.actamat.2010.05.057

[15] J. Čech, P. Haušild, O. Kovářík. Characterization of structural materials by spherical indentation. Mater Tehnol 51:695-698, 2017. DOI:10.17222/mit.2016.302 